\title{
Outpatient tubeless percutaneous nephrolithotomy and concomitant cystolitholapaxy
}

\author{
Kyle J. Lehmann, MD;* Darren Beiko, MD, MBA, FRCSC ${ }^{\dagger}$
}

*Department of Urology, Dalhousie University, Halifax, NS; 'Department of Urology, Queen's University, Kingston General Hospital, Kingston, ON

Cite as: Can Urol Assoc J 2014;8(3-4):e179-80. http://dx.doi.org/10.5489/cuaj.1675 Published online March 11, 2014.

\section{Abstract}

Percutaneous nephrolithotomy (PCNL) is a widely employed endourologic procedure to remove renal stones. Traditionally, PCNL has been performed on an inpatient basis, with patients routinely hospitalized postoperatively. Given the ongoing rising healthcare costs, a shift toward outpatient surgery is desirable. We report the case of a 21-year-old female who was safely discharged a few hours following uncomplicated tubeless PCNL and concomitant cystolitholapaxy for a stent with encrustation on its distal coil. To the best of our knowledge, this is the first published case of tubeless PCNL with concomitant cystolitholapaxy performed on an entirely outpatient basis.

\section{Introduction}

Percutaneous nephrolithotomy (PCNL) remains the gold standard in the surgical management of large renal calculi. Since its conception in $1976,{ }^{1}$ several innovations have been made to the procedure, including tubeless ${ }^{2}$ and totally tubeless $^{3}$ variants. These approaches have improved the surgical outcomes of $\mathrm{PCNL}$, reducing both postoperative pain and length of stay. ${ }^{2,4-6}$ Despite this, and despite the potential financial benefits to the healthcare system, few centres have explored the use of tubeless PCNL as an outpatient procedure..$^{7-9}$ To the best of our knowledge, there is only 1 published report on standard PCNL done on an outpatient basis, and this report involved 5 patients. ${ }^{10}$ Our report represents the first published case of tubeless PCNL with simultaneous cystolitholapaxy completed on an outpatient basis.

\section{Case report}

A healthy 21-year-old woman who was 36 weeks pregnant initially presented to the emergency room with left flank pain and fever. Physical examination and laboratory work were concerning for urosepsis, and the patient was started on antibiotics. Subsequent ultrasound revealed a large stone in the left renal pelvis, with mild hydronephrosis. A left ureteral stent was placed emergently and the patient was admitted to hospital for observation.

Following the uncomplicated delivery of her child, the patient was reassessed in clinic 2 weeks postpartum, at which time a computed tomography scan revealed a stable, $26 \times 12 \times 8$-mm left renal pelvic stone with no hydronephrosis. In addition, the previously placed ureteral stent displayed severe encrustation measuring $34 \times 20 \times 20 \mathrm{~mm}$ at the distal end, with minimal encrustation at the proximal end. Several endourologic treatment options-both combination and staged-were discussed with the patient. Given the size of the stone and the complex, encrusted stent, we recommended that the patient undergo a combination left PCNL and cystolitholapaxy. Informed consent was obtained.

Intraoperatively, standard antibiotic prophylaxis was administered and cystolitholapaxy was performed in the modified lithotomy position. Flexible cystourethroscopy revealed a normal urethra and a large bladder stone burden that represented the severely encrusted distal coil of the left ureteral stent. A 3-French electrohydraulic lithotripsy probe was used to perform complete lithotripsy of all encrusted material on the stent. Stone fragments were removed using a stone basket and Toomey syringe irrigation. Due to lack of proximal encrustation, the ureteral stent was removed without difficulty after placing a guidewire up the ureter and into the collecting system. The patient was then placed in the prone position for the PCNL part of the operation. Retrograde pyelogram revealed the large stone within the renal pelvis. Lower pole percutaneous access was achieved and the tract was dilated to a 30-French size using a balloon dilator. Using a 24-French rigid nephroscope, the stone was readily identified in the renal pelvis. Duckbill graspers were used to grasp the stone, and with subtle realignment of the stone along the axis of the sheath, the stone could just barely be removed intact. Blood loss was negligible throughout the entire procedure and therefore no nephrostomy tube was placed. A new $24-\mathrm{cm} \times 6$-French ureteral stent was inserted in an antegrade 
fashion. The total operative time was 105 minutes.

In the post-anesthetic care unit, the patient experienced minimal pain or hematuria. Her vital signs were stable and her hemoglobin 2 hours postoperative was 102; preoperatively it was 121 . The patient was discharged in stable condition 5.5 hours after leaving the operating room. Follow-up was arranged in clinic at 2 weeks. The patient was well, without any postoperative complications, and a kidneysureters-bladder (KUB) $\mathrm{x}$-ray showed no residual stones. The ureteral stent was removed cystoscopically. Stone analysis revealed the same mixed stone composition for the kidney and bladder; the majority consisted of calcium carbonate/ phosphate with magnesium ammonium phosphate and a minority was uric acid. Incidentally, the chemistry lab measured the renal stone to be $24 \times 10 \times 7 \mathrm{~mm}$, which explains why we were able to remove it intact through the 30 -French sheath. At the patient's 1-year follow-up, she remains well and stone-free by ultrasonography and KUB x-ray imaging.

\section{Discussion}

Since being described in 1976, the original PCNL procedure has undergone numerous modifications in an effort to reduce associated morbidity and mortality. Historically, nephrostomy tubes have been left in place following the procedure to tamponade bleeding and provide additional drainage. ${ }^{1}$ However, the presence of nephrostomy tubes is often associated with increased patient discomfort and prolonged hospitalization.

In 1997, Bellman and colleagues argued that tubeless PCNL reduced the postoperative analgesic requirement, length of hospital stay and overall cost as compared to the standard procedure. ${ }^{2}$ Since then, several randomized trials and literature reviews have confirmed the benefits of tubeless PCNL, and also revealed no significant differences in morbidity or stone-free rates between tubeless and standard approaches. ${ }^{11-14}$ Despite this, the adoption of tubeless, and especially outpatient PCNL, has been slow, and patients are still regularly admitted to hospital postoperatively. ${ }^{13}$

Few studies have published reports of tubeless or totally tubeless PCNL as an outpatient procedure..$^{7-9}$ In 2010, Shahrour and Andonian published their successful experience with ambulatory PCNL in a series of 10 carefully selected patients. ${ }^{7}$ They reported stone-free status in all patients, with a median hospital stay of 240 minutes and only 3 patients requiring home narcotic use. Thus, in appropriately selected patients, outpatient PCNL can be both safe and effective. Furthermore, the outpatient nature of the procedure carries additional benefits, including reallocation of healthcare resources, reduction in hospital costs and decreased risk of nosocomial complications.

In the present report, our patient met all of our previously published discharge criteria. ${ }^{9}$ The operation was uneventful, with minimal hemorrhage. Postoperatively, our patient did not have any significant pain, hematuria or flank leakage. Outpatient assessment revealed no complications following the operation and KUB x-ray and ultrasound confirmed a stone-free status.

\section{Conclusion}

The growing use of tubeless and totally tubeless PCNL has improved patient outcomes and challenged the need for routine postoperative hospitalization. In the current report, we present the first published case of tubeless outpatient PCNL with concomitant cystolitholapaxy. Additional studies in a larger patient population are warranted to better characterize the efficacy and safety of outpatient PCNL on a broader scale.

Competing interests: Dr. Lehmann and Dr. Beiko declare no competing financial or personal interests.

This paper has been peer-reviewed.

\section{References}

1. Fernström I, Johansson B. Percutaneous pyelolithotomy: A new extraction technique. Scand I Urol Nephrol 1976;10:257-9.

2. Bellman GC, Davidoff R, Candela J, et al. Tubeless percutaneous renal surgery. J Urol 1997; 157:1578-82.

3. Aghamir SM, Hosseini SR, Gooran S. Totally tubeless percutaneous nephrolithotomy. J Endourol 2004; 18:647-8.

4. Singh I, Singh A, Mittal G. Tubeless percutaneous nephrolithotomy: Is it really less morbid? J Endourol 2008;22:427-34. http://dx.doi.org/10.1089/end.2007.0269

5. Yuan $H$, Zheng $S$, Liu L, et al. The efficacy and safety of tubeless percutaneous nephrolithotomy: a systematic review and meta-analysis. Urol Res 2011;39:401-10. http://dx.doi.org/10.1007/s00240010-0355-5

6. Amer T, Ahmed K, Bultitude M, et al. Standard versus tubeless percutaneous nephrolithotomy: A systematic review. Urol Int 2012;88:373-82. http://dx.doi.org/10.1159/000336145

7. Shahrour W, Andonian S. Ambulatory percutaneous nephrolithotomy: Initial series. Urology 2010;76:128892. http://dx.doi.org/10.1016/i.urology.2010.08.001

8. Beiko D, Samant M, McGregor TB. Totally tubeless outpatient percutaneous nephrolithotomy: initial case report. Adv Urol 2009;295825:3 pages. http://dx.doi.org/10.1155/2009/295825

9. Beiko D, Lee L. Outpatient tubeless percutaneous nephrolithotomy: The initial case series. Can Urol Assoc J 2010;4:E86-90.

10. Preminger GM, Clayman RV, Curry T, et al. Outpatient percutaneous nephrostolithotomy. J Urol 1986; 136:355-7.

11. Chang $\mathrm{CH}$, Wang $\mathrm{CJ}$, Huang SW. Totally tubeless percutaneous nephrolithotomy: A prospective randomized controlled study. Urol Res 2011;39:459-65. http://dx.doi.org/10.1007/s00240-011-0363-0

12. Ni S, Qiyin C, Too W, et al. Tubeless percutaneous nephrolithotomy is associated with less pain and shorter hospitalization compared with standard or small bore drainage: A meta-analysis of randomized, controlled trials. Urology 2011;77:1293-8. http://dx.doi.org/10.1016/j.urology.2010.10.023

13. Agrawal MS, Agrawal M, Gupta A, et al. A randomized comparison of tubeless and standard percutaneous nephrolithotomy. J Endourol 2008;22:439-42. http://dx.doi.org/10.1089/end.2007.0118

14. Shah $H$, Khandkar A, Sodha $H$, et al. Tubeless percutaneous nephrolithotomy: 3 years of experience with 454 patients. BJU Int 2009;104:840-6. http://dx.doi.org/10.1111/i.1464-410X.2009.08496.x

Correspondence: Dr. Darren Beiko, Associate Professor, Department of Urology, Queen's University, Kingston General Hospital, 76 Stuart St., Kingston, ON K7L 2V7; fax: 613-545-1970; beikod@kgh.kari.net 